\title{
L'HOMME L'Homme
}

$229 \mid 2019$

Varia

\section{Raymond Jamous, Le Sultan des frontières. Essai d'ethnologie historique du Maroc}

Charles Malamoud

\section{(2) OpenEdition}

1 Journals

Édition électronique

URL : https://journals.openedition.org//homme/33691

DOI : 10.4000//homme.33691

ISSN : 1953-8103

Éditeur

Éditions de l'EHESS

\section{Édition imprimée}

Date de publication : 1 mars 2019

Pagination : 188-190

ISSN : 0439-4216

\section{Référence électronique}

Charles Malamoud, «Raymond Jamous, Le Sultan des frontières. Essai d'ethnologie historique du

Maroc », L'Homme [En ligne], 229 | 2019, mis en ligne le 01 mars 2019, consulté le 08 janvier 2022

URL : http://journals.openedition.org//homme/33691; DOI : https://doi.org/10.4000//homme.33691 


\section{Raymond Jamous}

Le Sultan des frontières. Essai d'ethnologie historique du Maroc

Nanterre, Société d'ethnologie, 20I7, I60 p., bibl., gloss., ill.

(«Maghreb et Mashreq»).

L E PETIT livre que propose Raymond Jamous, à la fois dense, pénétrant et lumineux, porte sur la nature de la royauté marocaine. Cette enquête relève, en premier lieu, de l'histoire. Elle est suscitée par une interrogation, ou plutôt un étonnement: «Le Maroc est le seul pays de l'Afrique du Nord et du Proche-Orient arabe qui ait connu un sultanat depuis un millénaire et n'ait subi d'occupation coloniale que pour une courte période, au xx siècle» (p. 9). Et il faut rappeler que l'expansion ottomane s'est arrêtée au Maroc.

Que s'est-il passé, que s'est-il construit, au cours des siècles, qui explique cette pérennité? Il importe de préciser, pour donner sens à cette question, que le sultanat s'est profondément modifié, au XVI ${ }^{\mathrm{e}}$ siècle, avec l'accession au pouvoir de dynasties de chorfa, descendants du Prophète et, plus particulièrement au XVII ${ }^{e}$, de la dynastie chérifienne des Alaouites, qui règne encore de nos jours. L'enquête historique, dans la démarche de Raymond Jamous, rencontre la théorie politique: comment caractériser les relations entre le sultan et ses sujets aux différentes phases de l'histoire de la monarchie marocaine? L'auteur prend appui sur l'œuvre du Marocain Ibn Khaldoun, qui vivait au XIV ${ }^{e}$ siècle, pour la période pré-chérifienne, et sur la pensée de Tocqueville, pour ce qui est des «fondements d'une sociologie comparative» (pp. 91-93).

Mais ce qui singularise ce travail, et en fait tout le prix, c'est que les données his- 
toriques et les généralisations conceptuelles sont abordées ici par un ethnologue qui a fait du Maroc le terrain privilégié de son expérience et de sa réflexion. Il faut rappeler, en effet, que Raymond Jamous est l'auteur de Honneur et baraka', fruit d'une longue étude de terrain, dans le Rif, et que le livre qu'il présente aujourd'hui en est, d'une certaine manière, le prolongement. C'est donc en pleine conscience des difficultés et des enjeux que l'ethnologue qu'il est réfléchit sur la possibilité de faire une analyse ethnologique de données recueillies autrefois par des auteurs que nous ne pouvons que lire, et qui n'étaient pas eux-mêmes ethnologues. Raymond Jamous affirme, et prouve, qu'en l'occurrence, tout au moins, cette démarche est légitime et féconde.

Lessentiel de ce travail, en effet, est une enquête sur une pratique remarquable des sultans alaouites, dont la dernière manifestation a eu lieu en 1894 , et que nous ne connaissons donc que par les descriptions, souvent très détaillées, de témoins aujourd'hui disparus. Cette pratique est celle des expéditions de grande ampleur, très spectaculaires, que le sultan menait régulièrement vers les tribus qui occupaient les différentes régions du royaume. Raymond Jamous insiste, comme il se doit, sur les aspects rituels et le symbolisme de ces expéditions, dont la finalité est au premier abord politique: il s'agit d'affirmer, de confirmer, la relation, infiniment complexe, entre le roi et ses sujets. Le sultan alaouite, descendant du Prophète, est doué de baraka, pouvoir de bénédiction et de sanctification, source de vie. Les expéditions du sultan ont certes pour but de manifester sa souveraineté politique et militaire, et donc, le cas échéant, de rétablir l'ordre, prévenir ou réprimer des révoltes, mais leur raison d'être primordiale est de déployer et de faire reconnaître la puissance de sa baraka. Or, le sultan n'est pas seul à être doué de baraka. Il partage ce pouvoir avec des "saints" locaux, vivants ou morts, eux aussi descendants du Prophète, autour desquels s'organisent des confréries influentes dans les tribus que le sultan vient visiter. Le paradoxe est que le sultan n'est vraiment souverain que s'il a été reconnu solennellement et rituellement comme tel par les détenteurs locaux de la baraka. Il n'entre pas dans le territoire des tribus, mais s'installe à la frontière: de sorte que c'est lui, cet étranger venu d'ailleurs, qui offre son hospitalité, en même temps que sa justice et sa baraka, aux populations qui viennent le visiter comme on visite le sanctuaire d'un saint. Les itinéraires du sultan suivent donc toujours des frontières. Loin de se réduire à des limites plus ou moins franchissables, les frontières, en l'occurrence, sont des espaces que le sultan parcourt et occupe lors de ses expéditions, et c'est à partir de ces limites entre les différentes parties du royaume qu'il affirme sa souveraineté sur l'ensemble.

En ce sens, le sultan est un souverain «en relation » et c'est par là que se justifie le titre de l'ouvrage. Le lien entre la royauté et la frontière ainsi conçue est si puissant que, même quand le sultan est dans sa capitale, il installe, pour célébrer le sacrifice de l'aïd, une réplique du campement mobile qui est sa résidence quand il est en expédition. Il faut aussi noter que, dans ses itinérances, le sultan met ses pas dans les pas de ses prédécesseurs et que le lien spatial qu'il établit avec ses sujets se double ainsi d'un lien temporel avec les générations passées. Le rituel si exigeant du voyage dans les frontières et le jeu si complexe des ajustements de baraka sont, si on comprend bien, ce qui fait la force de la relation entre le sultan et ses sujets, et ce qui explique la remarquable pérennité du royaume. Par définition commandeur des croyants, le sultan ne cherche pas à étendre son territoire, mais à tenir ensemble les différentes parties du domaine où s'exerce sa baraka.

Cet ouvrage de Raymond Jamous, bien que bref, est d'une richesse foisonnante. Il faut en souligner la portée théorique. Il est une contribution précieuse aux réflexions qui se développent actuellement parmi

1. Cf. Raymond Jamous, Honneur et baraka. Les structures sociales traditionnelles dans le Rif, Cambridge-New York, Cambridge University Press / Paris, Éd. de la Msh, 1981 («Atelier d'anthropologie sociale»). 
les anthropologues, les géographes, les politiques et les juristes sur la notion de frontière. La «frontière" (dont on notera au passage qu'elle ne fait pas l'objet, pour le moment, d'une entrée dans le glossaire qui accompagne le livre) n'est donc pas ici la limite qui entoure un territoire et que le souverain pourrait avoir l'ambition de repousser indéfiniment (comme c'est le cas pour le jihad conquérant des dynasties qui ont précédé les chorfa), c'est un espace que le centre occupe et parcourt pour que les parties qui constituent le territoire forment un tout articulé.

Charles Malamoud 